\title{
Error Rate Estimation Based on Soft Output Decoding and its Application to Turbo Coding
}

\author{
Emilio Calvanese Strinati, Sébastien Simoens and Joseph Boutros \\ Email: emilio.calvanese-strinati@cea.fr, simoens@motorola.com, boutros@enst.fr
}

\begin{abstract}
In this paper, we investigate reliable error rate estimation techniques for MAC layer adaptive mechanisms. In particular, we propose and analyze a novel error rate estimator based on soft output information available as output of receivers with turbo principle. Contrary to previous works on the subject, we relax the assumption of perfect knowledge of Signal-Noiseto-Ratio (SNR) at the receiver, and we analyze the impact of a SNR estimation error on the error rate estimate. We show that, differently to previous techniques, the proposed estimation method is insensitive to such SNR estimation error. Our analytical and simulation results validate the conclusion.
\end{abstract}

Index Terms-Soft-output decoding, channel state estimation.

\section{INTRODUCTION}

New generations of broadband wireless systems employ advanced coding combined with reliable radio link control techniques to optimize the use of limited available resources bandwidth, power, etc. The concept behind radio link control techniques, such as Link Adaptation (LA), is to take advantage of a link as efficiently as possible in given channel conditions by adjusting certain transmission parameters. This adaptation relies on performance prediction that is derived from crosslayer information between the physical and the MAC layers. Adaptation is not an easy task. In Orthogonal Frequency Division Multiplexing systems (OFDM), the frequency-selective channel created by multipath propagation is turned into parallel flat fading channels, where different subcarriers experience different fading. This fading is often time-varying due to user and environment mobility. Moreover, the channel may be subject to time-varying interference. In such context,it is of high interest to further improve error prediction methods. In order to guarantee the Quality of Service (QoS) constraints, adaptive mechanisms implement a suboptimal trade-off between link robustness and bandwidth efficiency. In [1], we benchmarked soft-output based error prediction with other error rate prediction techniques by computing the throughput performance of an Adaptive Modulation and Coding (AMC) algorithm with QoS constraints in n existing OFDM system (IEEE 802.11a). Here in this paper, we detail the theoretical derivation of these soft-output based error estimators, and

Emilio Calvanese Strinati is with CEA/LETI Labs, Grenoble, France and with ENST, Paris, France. Sébastien Simoens is with Motorola Labs, Paris, France. Joseph Boutros is with ENST, Paris, France. investigate some properties related to turbo-codes design, such as interleaver size. Our work is inspired by three interesting results on decoders with turbo principle [2], [3].

First, it has been shown that a priori information on the decoded bits can be used to evaluate the error rate faster than the classic Monte Carlo simulation method [4], [5], [6]. Likewise, in [7] a BER estimator is proposed based on an estimate of the log likelihood ratios (LLR) distribution. In [8] the mean and variance of LLR are computed, in order to derive an ML estimator imposing a symmetry assumption on the LLR probability distribution. All those BER estimators are derived by computing the expectation of LLR (or of a function of it) assuming that the LLR are Gaussian distributed and that the SNR is known to the decoder. However, experimental data suggest that these outputs are not Gaussian distributed [9]. There are techniques for BER estimation based on maximum entropy methods that do not require a Gaussian assumption [10].

Second, several authors proposed to accelerate the iterative decoding and reduce the complexity of a receiver with turbo principle by dynamically controlling the number of decoding iterations on a packet-by-packet basis through a properly defined stopping rule. One of the first papers on stopping criteria is [11], which is based on cross entropy (CE) between the output distributions of the different decoder modules. Based on the same CE concept, Shao et al. [12] introduced two simple methods called sign-change-ratio (SCR) criterion and hard-decision aided (HDA) criterion. In [13] , extending the SCR method, a new criterion named sign difference ratio (SDR) is proposed. It uses the sign differences between a priori information and extrinsic information. The method presented by Zhai and Fair in [14], which they called the mean estimate (ME), is to compare the mean of the output LLRs values with a pre-defined threshold. In [15] Scavino et al. propose a novel stopping criterion based on the average entropy of an information block, which is a measure of the reliability of bit decisions, for an information block of a given size. More work on stopping criteria can be found in [16], [17], [18].

Third, recent studies investigate the sensitivity of soft output decoders (MAP and max-log-MAP) to an SNR estimation error. In [19] it is shown that while MAP and log-MAP decoders need a priori knowledge of noise variance to correctly estimate the a posteriori probability of the decoded bits, 
max-log-MAP decoders are totally insensitive to such error. However, in it is shown that MAP and log-MAP decoders can tolerate a significant SNR estimation error without error rate degradation [20], [21]. Nevertheless, the expectation of LLR strongly depends on the SNR estimation error also if error rate is not.

Merging together these three properties of iterative decoders, we derive a new error rate estimator which is also based on the LLR distribution, but which does not exhibit dependence on the SNR estimation errors. We quantify the impact of SNR estimation error on LLR based estimators by analytical calculations for uncoded transmission, as well as by computer simulations for both convolutionally and turbo coded transmission.

We first introduce essential notations and theoretical background on soft-output decoding information available at the output of a receiver with turbo principle in section II. In section III, we proceed to analytically examine two error rate estimation methods based on the use of soft outputs in the case of uncoded BPSK transmission over an AWGN channel. In section IV, we extend our analytic results to Convolutional/turbo coded transmission for the AWGN channel by means of numerical simulation. Finally, section $\mathrm{V}$ concludes the paper.

\section{THEORETICAL BACKGROUND}

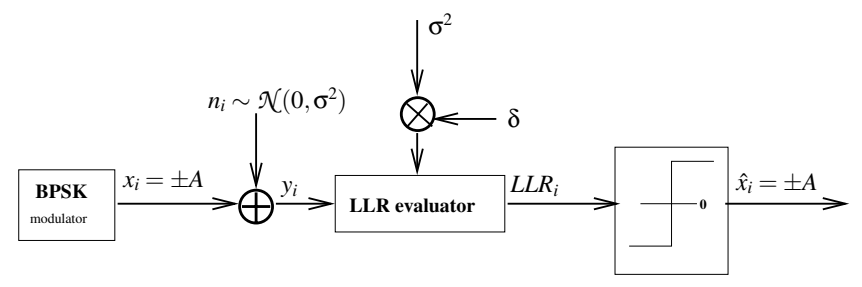

Fig. 1. Transmission path model.

In systems were a soft output decoder is adopted, the Log Likelihood Ratio (LLR) probability of the transmitted bits are available as output of the decoder. Consider the transmission path of Fig. 1, where $x_{i}= \pm A$ is the transmitted symbol, $n_{i}$ the additive white Gaussian noise of variance $\sigma^{2}=\frac{N_{0}}{2}$, and $y_{i}$ the received signal. We assume perfect signal amplitude estimation at the receiver and, consider a relative estimation error on $\sigma^{2}$ by introducing the scaling factor $\delta$. We indicate with $L L R_{i}$ the random variable following the distribution (1) related to the i-th transmitted symbol $x_{i}$ of the packet. If bits are modulated using a BPSK modulation over an AWGN channel, the probability distribution of $L L R_{i}$ is given by:

$$
p_{L L R}\left(L L R_{i}\right)=p[x=-A] \mathcal{N}\left(-\frac{\alpha}{\delta}, \frac{\beta^{2}}{\delta^{2}}\right)+p[x=+A] \mathcal{N}\left(\frac{\alpha}{\delta}, \frac{\beta^{2}}{\delta^{2}}\right)
$$

where $\mathcal{N}\left(m, \sigma^{2}\right)$ denotes the normal distribution of mean $m$ and variance $\sigma^{2}$, and $\beta^{2}=2 \alpha=\frac{4 A^{2}}{\sigma^{2}}$. Under the above hypothesis, the BER of the uncoded transmission $\left(B E R_{\text {uncoded }}\right)$ equals:

$$
B E R_{\text {uncoded }}=Q\left(\frac{A}{\sigma}\right)
$$

with $Q(x)=\frac{1}{\sqrt{2 \pi}} \int_{x}^{\infty} e^{-\frac{u^{2}}{2}} d u$. The BER is indeed univocally identified by the ratio $A / \sigma=\alpha / \beta$. The parameters $\alpha$ and $\beta$ are not obviously estimated from $p_{L L R}\left(L L R_{i}\right)$, since the tails of the two Gaussian distributions overlap. Furthermore, the statistical moments of the LLR distribution depend on both $\frac{A}{\sigma}$ and $\delta$. Moreover, while the statistical moments of the LLR distribution depend on both $\frac{A}{\sigma}$ and $\delta$, the BER may not satisfy

$$
B E R\left(\frac{A}{\sigma}, \delta\right) \simeq B E R\left(\frac{A}{\sigma}, 1\right) .
$$

\section{BER ESTIMATION}

In this section we present two error rate estimation methods derived as function of the statistics of a posteriori LLR at the receiver output. We analyze the bias of the two estimation methods in the context of uncoded BPSK or QPSK modulated symbols transmitted over the AWGN channel, for which formula (1) holds. We show that the first method, already known in the literature, depends on SNR uncertainty $\delta$. Then, we propose a new method, Method 2, insensitive to $\delta$.

\section{A. Method 1}

A simple BER estimator can be directly obtained at each decoded packet using the A Posteriori Probabilities (APP) available after Soft Output decoding of the received packet. Considering the transmission path of figure 1 , let $\mathrm{N}$ be the packet length, denoting with $A P P_{i}(+A)$ and $A P P_{i}(-A)$ the $\mathrm{A}$ Posteriori Probabilities of the i-th transmitted symbol $x_{i}$ of the packet then, a BER estimator can be formalized as follows:

$$
B \hat{E} R_{1}=\frac{1}{N} \cdot \sum_{i=1}^{N} \min \left(A P P_{i}(-A), A P P_{i}(+A)\right)
$$

In [4], [5] the equivalent BER estimator was proposed to accelerate the Monte Carlo simulation:

$$
B \hat{E} R_{1}=\frac{1}{N} \cdot \sum_{i=1}^{N} \frac{1}{1+e^{\left|L L R_{i}\right|}}
$$

Where formula (4) and (5) are two expression of the same equation since:

$$
A P P(+A)=\frac{1}{1+e^{-L L R}}
$$

and

$$
A P P(-A)=\frac{1}{1+e^{L L R}}
$$

We compute the $E\left[B \hat{E} R_{1}\right]$ (where $B \hat{E} R_{1}$ is given by formula (5)) for an uncoded BPSK or QPSK modulation in order to study the bias of the estimator. Contrary to prior art 
we consider the presence of an estimation error $\delta$ on the channel noise variance. For simplification, we provide the demonstration only for a BPSK modulation:

$$
\begin{aligned}
E\left[B \hat{E} R_{1}\right] & =E\left[\frac{1}{1+e^{|L L R|}}\right]=\int_{-\infty}^{+\infty} \frac{1}{1+e^{|t|}} \cdot p_{L L R}(t) d t \\
= & \frac{1}{2} \cdot[\underbrace{\int_{-\infty}^{0} \frac{1}{1+e^{-t}} \cdot \mathcal{N}\left(\frac{\alpha}{\delta}, \frac{\beta^{2}}{\delta^{2}}\right) d t}_{B E R-I_{2}}+\underbrace{\int_{0}^{\infty} \frac{1}{1+e^{t}} \cdot \mathcal{N}\left(\frac{-\alpha}{\delta}, \frac{\beta^{2}}{\delta^{2}}\right) d t}_{B E R-I_{2}} \\
& +\underbrace{\int_{-\infty}^{0} \frac{1}{1+e^{-t}} \cdot \mathcal{N}\left(\frac{-\alpha}{\delta}, \frac{\beta^{2}}{\delta^{2}}\right) d t}_{I_{1}}+\underbrace{\int_{0}^{\infty} \frac{1}{1+e^{t}} \cdot \mathcal{N}\left(\frac{\alpha}{\delta}, \frac{\beta^{2}}{\delta^{2}}\right) d t}_{I_{1}}] \\
= & B E R+I_{1}-I_{2}
\end{aligned}
$$

where:

$$
I_{1}=\int_{0}^{\infty} \frac{1}{1+e^{t}} \cdot \frac{\delta}{\beta \cdot \sqrt{2 \pi}} \cdot e^{-\frac{\left(t-\frac{\alpha}{\delta}\right)^{2}}{2 \frac{\beta^{2}}{\delta^{2}}}} d t
$$

and

$$
I_{2}=\int_{0}^{\infty} \frac{e^{t}}{1+e^{t}} \cdot \frac{\delta}{\beta \cdot \sqrt{2 \pi}} \cdot e^{-\frac{\left(t+\frac{\alpha}{\delta}\right)^{2}}{2 \frac{\beta^{2}}{\delta^{2}}}} d t
$$

Finally, the bias of the estimator can be expressed as:

$$
I_{1}-I_{2}=\frac{\delta}{\beta \cdot \sqrt{2 \pi}} \cdot \int_{0}^{\infty} \frac{e^{-\frac{\left(t^{2} \delta^{2}+\alpha^{2}\right)}{2 \beta^{2}}}}{1+e^{t}} \cdot\left[e^{\frac{\delta t}{2}}-e^{\left(1-\frac{\delta}{2}\right) t}\right] \cdot d t
$$

then, $I_{1}-I_{2} \geq 0$ if $t(\delta-1) \geq 0$. Indeed, since $t \in[0,+\infty]$ :

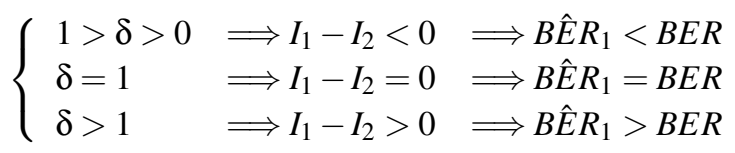

Hence, from conditions (7) we evict that if SNR is overestimated, BER is underestimated and vice versa, if SNR is underestimated, BER is overestimated. Consequently, $B \hat{E} R_{1}$ is unbiased for uncoded BPSK or QPSK modulations only if $\delta=1$. Simulation results (see section IV) will extend the validity of this result for both convolutionally and turbo coded case.

\section{B. Method 2}

From formula (2) we know that the BER of uncoded BPSK transmitted symbols is univocally determined by the ratio $\alpha / \beta$, that is the mean to standard deviation ratio. From formula (3) we infer that under certain conditions ${ }^{1}$ also the BER after decoding is insensitive to such noise variance estimation error. As consequence it should exist a class of error rate estimators that should be totally insensitive to $\delta$ and we aim to find one method of this class. We showed in section III-A how the error estimation method defined by equation (5) is biased with $\delta$.

\footnotetext{
${ }^{1}$ when the decoder is a MAP or a log-MAP formula (3) holds if $\delta \in[-2,+6]$ $\mathrm{dB}$ [20], [21], otherwise if the decoder is a max-log-MAP formula (3) holds $\forall \delta$ [19].
}

This is due to the fact that in (5) we try to estimate the error rate computing a function of the mean of the $L L R_{i}$, while such mean varies with $\delta$.

Assuming that the LLR distribution after decoding are approximately Gaussian ${ }^{2}$, the BER of coded BPSK transmitted symbols should be univocally determined by the ratio $\alpha / \beta$ of the decoder output LLR distribution. Unfortunately $\alpha / \beta$ after decoding is unknown. We propose to estimate $\alpha / \beta$ by computing the mean to standard deviation ratio of $\left|L L R_{i}\right|$ at the output of the decoder with turbo principle. In the following we will call this ratio $\Lambda$. Considering the same transmission path of section III-A, $\Lambda$ can be computed at each packet observation computing the mean and standard deviation of | $L L R$ | from the observation of the $L L R_{i}$ :

$$
\Lambda=\frac{E[|L L R|]}{\sqrt{E\left[(|L L R|-E[|L L R|])^{2}\right]}}
$$

Actually, mean and standard deviation of | $L L R \mid$ over the $\mathrm{N}$ observed symbols can be computed either directly or by first computing a histogram of the LLR observed values. The second approach results typically in a smoother estimation, and it may return better results for the estimation of mean and standard deviation, but this is not the scope of this section. Moreover, for small values of BER, the impact of the overlapping of the two normal distributions in (1) becomes negligible, and we have $\Lambda \approx \frac{\alpha}{\beta}$. Such a situation corresponds to a typical operational context since the target BER of an adaptive mechanism is often below $10^{-4}$.

Then, the proposed estimator is defined as:

$$
B \hat{E} R_{2}=h(\Lambda)
$$

where $h(\Lambda)$ is a bijective function that univocally links the BER to $\Lambda$ defined by equation (8). For uncoded BPSK modulation transmitted over an AWGN channel, the LLR distribution is provided in (1) and (8) and can be expressed as follows (algebraic manipulations are omitted):

$$
\Lambda=\frac{\sqrt{2} \cdot\left(-\frac{1}{2}+Q\left(\frac{\alpha}{\beta}\right)-\frac{1}{\sqrt{2 \pi} \cdot \frac{\alpha}{\beta}} \cdot e^{-\frac{1}{2} \cdot\left(\frac{\alpha}{\beta}\right)^{2}}\right)}{\sqrt{\left(\frac{1+\left(\frac{\beta}{\alpha}\right)^{2}}{2}\right)-2 \cdot\left(-\frac{1}{2}+Q\left(\frac{\alpha}{\beta}\right)-\frac{1}{\sqrt{2 \pi} \cdot \frac{\alpha}{\beta}} \cdot e^{-\frac{1}{2} \cdot\left(\frac{\alpha}{\beta}\right)^{2}}\right)^{2}}}
$$

Studying the expression (10) we infer that $\Lambda$ is monotonically decreasing depending only on the ratio $\frac{\alpha}{\beta}$ at a given modulation and coding scheme. Consequently, so does $B \hat{E} R_{2}$, being independent from $\delta$. The function $h(\Lambda)$ can be implemented by means of a Look Up Table (LUT) which can be easily obtained analytically since both $\Lambda$ and BER are functions of the same ratio $\frac{\alpha}{\beta}$. The LUT of (9) can be easily computed from (10), since $\frac{\alpha}{\beta}=\frac{A}{\sigma}$ and $B E R=Q\left(\frac{A}{\sigma}\right)$.

\footnotetext{
${ }^{2}$ It was observed in [22] that the pdf of the $L L R_{i}$ after decoding is nearly Gaussian
} 


\section{Simulation Results}

In this section the two BER estimation methods (5) and (8) are compared in terms of $B \hat{E} R$ versus $\frac{E b}{N 0}$ in the AWGN context. The transmission path is illustrated on Fig. 1. Results for uncoded transmission are reported on Fig. 2, on Fig. 3 for Convolutionally-coded transmission and on Fig. 4 and 5 for Turbo-Coded transmission in the AWGN. We verify that both methods are robust against code parameters but we present here simulation results only for the following code design.

For uncoded transmission the probability distribution of the LLR at the output of the APP computer is given by (1). Therefore, in section III we derived mathematically the bias for Method 1 and the analytical expression of $\Lambda$ (equation (10)).

On Fig. 2, when $\delta=1$ (i.e., perfect knowledge of the channel noise variance at the receiver), both methods return an unbiased estimation of BER. On the contrary, when $\delta \neq 1$, only Method 2 is unbiased. Method 1 over-estimates the BER for $\delta>1$ and under-estimates it for $0<\delta<1$, as predicted by (7).

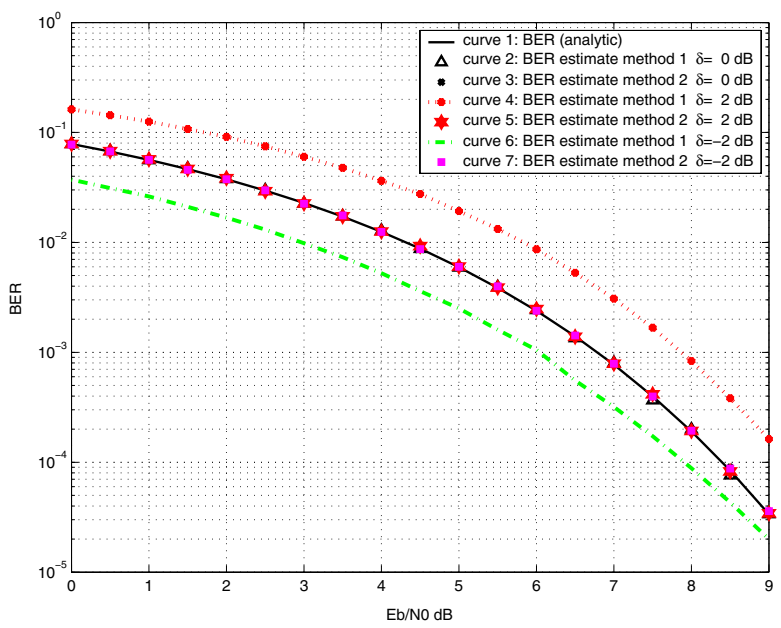

Fig. 2. Uncoded transmission: comparison of Method 1 and Method 2 with $\delta=0 \mathrm{~dB}$ and $\pm 2 \mathrm{~dB}$. Curves $1,23,5,7$ are identical

Since in case of coded transmission a mathematical expression for the probability distribution of the LLR at the output of the Soft Output decoder is unknown, in Fig. 3 the two estimators are compared by means of Monte Carlo simulation in the case of Convolutionally-coded transmission. The error-correcting code is the classical rate 1/2 64-state $(133,171)_{8}$ Convolutional code as defined in the IEEE 802.11 standard and the decoder is a log-MAP). We observe the same behavior as for uncoded transmission. For a given value of BER, Method 1 presents an over-estimation of $0.5 \mathrm{~dB}$ in terms of $\frac{E b}{N 0}$ when $\delta=2 d B$ and less than $0.5 \mathrm{~dB}$ of under-estimation when $\delta=-2 d B$.

Since soft output decoding is usually employed in receiver with turbo principle we analyze hereafter the performance

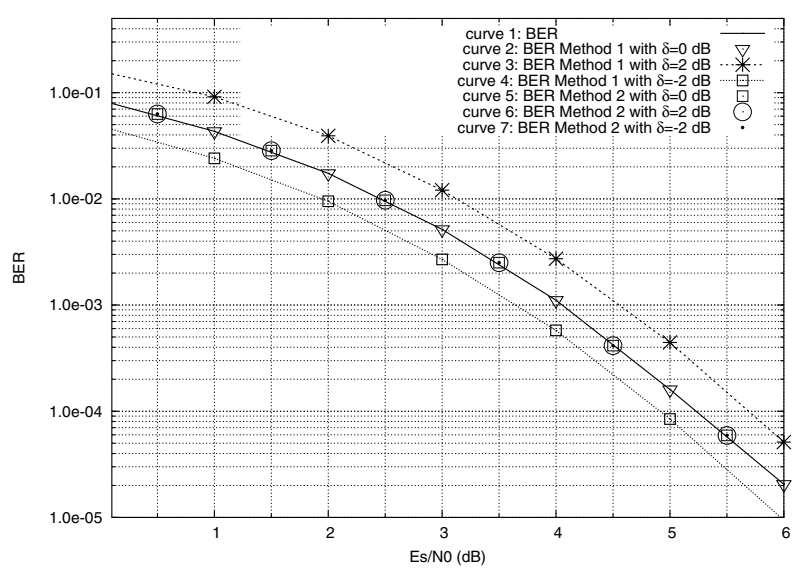

Fig. 3. Convolutionally-coded transmission: comparison of Method 1 and Method 2 with $\delta=0 \mathrm{~dB}$ and $\pm 2 \mathrm{~dB}$. Curves $1,2,5,6,7$ are identical.

of the two BER estimation methods for turbo receivers. We first compare them when $\delta=0 d B$. In Method 1, equation (5) requires the output $L L R_{i}$ values at the last decoding iteration. Nevertheless, while the extrinsic information exchanged between the two constituent Convolutional codes are assumed to be independent in Method 1, so it is only if the interleaver size is infinite. In practice the interleaver is of finite size. In such a case, Method 1 underestimates the BER due to the optimistic $L L R_{i}$ values assumed by the estimator. The smaller the interleaver size is, the more this phenomenon is remarkable.

In Fig. 4 a rate $1 / 3$ Turbo code with 4-state $\operatorname{RSC}(7,5)$ constituents is considered. Under perfect knowledge of the SNR, the performance of Method 1 have been analyzed for interleaver sizes of 1024, 4096 and 16000. The simulation results confirm that Method 1 applied to the turbo principle is biased also for $\delta=0 d B$ and that the experienced bias fades with increasing interleaver size.

In Method 2 the bias presented by Method 1 when the information block size is finite can be directly avoided defining the LUT.

In Fig. 5 a rate $1 / 3$ Turbo code with 4-state $\operatorname{RSC}(7,5)$ constituents is decoded with a max-log-MAP decoder. The information block length is 1024, the number of decoding iterations is limited to 12 and $\delta=\{0 \mathrm{~dB},-2 \mathrm{~dB},-3 \mathrm{~dB}\}$. Our numerical results show how, when $\delta=0$ only Method 2 returns an unbiased estimation of BER albeit the finite interleaver size. Moreover, when $\delta \neq 0$, while Method 1 present a greater bias, Method 2 is still unbiased. Note that our proposed method can be applied to the coded case for which the LLR distribution is not exactly Gaussian, since it only requires function $h(\Lambda)$ in equation (9) to be bijective. This assumption is confirmed by simulation for convolutionally and turbo coded transmission in which the LUT $h(\Lambda)$ is obtained by simulation. 


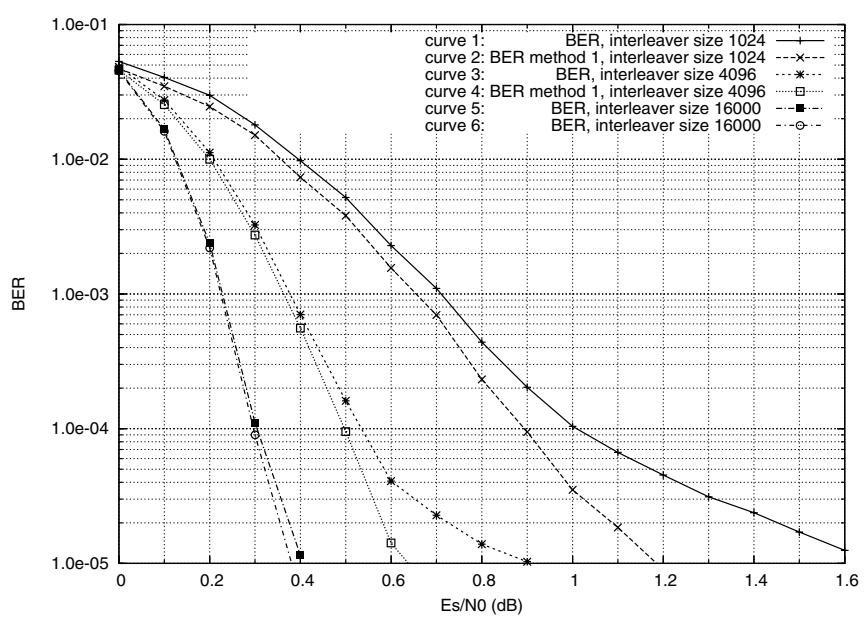

Fig. 4. Turbo coded transmission: Method 1 with $\delta=0 \mathrm{~dB}$.

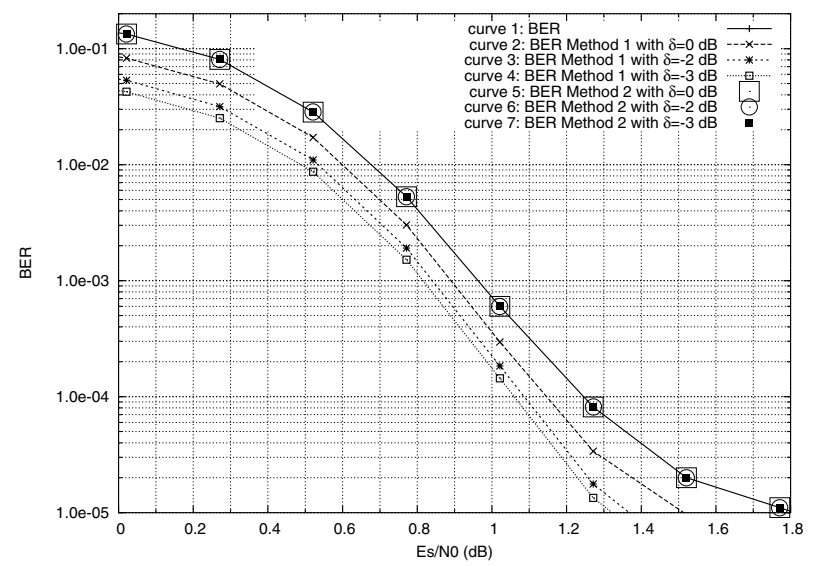

Fig. 5. Turbo coded transmission: comparison of Method 1 and Method 2 with $\delta=0,-2,-3 \mathrm{~dB}$. Curves $1,5,6,7$ are identical.

\section{CONCLUSions}

In this paper we use soft output decoding information to derive a reliable error rate estimation method for trellis coded symbols transmission over AWGN channel. Then, considering antipodal uncoded AWGN symbol transmission, we show analytically that while method 1 is unbiased only if SNR is correctly estimated, the proposed method 2 is independent from a SNR estimation error. We confirm these results by simulation for convolutionally/turbo coded transmission in the AWGN channel in both cases of perfect and mismatched noise variance SNR estimation. Contrary to previous techniques, the proposed method is independent from both noise variance estimation error and finite information block length.

\section{REFERENCES}

[1] E. Calvanese Strinati and S. Simoens and J. Boutros, "New Error Prediction Techniques for Turbo-Coded OFDM Systems and Impact on Adaptive Modulation and Coding," IEEE International Symposium on Personal, Indoor, and Mobile Radio Communications , Berlin, September 2005.

[2] L. Bahl and J. Cocke and F. Jelinek and J. Raviv, "Optimal Decoding of Linear Codes for Minimizing Symbol Error Rate," IEEE Transactions on Information Theory, vol. 20, pp. 284-287, March 1974.

[3] C. Berrou and A. Glavieux and P. Thitimajshima, "Near Shannon limits error-correcting coding and decoding: turbo-codes," IEEE Information conference on Communication, pp. 1064-1700, May 1993.

[4] H.A. Loeliger, "A posteriori probabilities and performance evaluation of trellis codes," IEEE International Symposium on Information Theory, Trondheim, June 1994.

[5] I. Land and P.A. Hoeher, "Log-Likelihood values and Monte Carlo simulation - some fundamental results " International Symposium on on Turbo Codes \& Related Topics, Brest, September 2000.

[6] I. Land and P.A. Hoeher, "New results on Monte Carlo bit error simulation based on the a posteriori log-likelihood ratio," International Symposium on on Turbo Codes \& Related Topics, Brest, September 2003.

[7] FITNESS, "Performance analysis of re-configurable MTMR transceivers for WLAN," http://www.ist-fitness.org/, 2002.

[8] N. Letzepis and A. Grant, "Bit error rate estimation for turbo decoding," Australian Communications Theory Workshop, Melbourne, February 2003.

[9] N. Letzepis and A. Grant "Non-Gaussian Behaviour of Extrinsic Log-Values," IEEE International Symposium on Information Theory, Chicago, June 2004.

[10] A. Abedi , "Invariance Properties and Performance Evaluation of Bit Decoding Algorithms," $\mathrm{PhD}$ thesis, University of Waterloo, Ontario, Canada, 2004.

[11] J. Hagenauer and E. Offer and L. Papke, "Iterative Decoding of Binary Block and Convolutional Codes," IEEE Transaction on Information Theory, vol. 42, pp. 429-445, March 1996.

[12] R.Y. Shao and S. Lin and M.P.C. Fossorier, "Two Simple stopping criteria for turbo decoding," IEEE Transaction on Communications, vol. 47, pp. 117-120, August 1999.

[13] Y. Wu and B. D. Woerner and W.J. Ebel, "A simple stopping criterion for turbo decoding," IEEE Communication Letters, Vol. 46, pp 421-423, August 2000.

[14] F. Zhai and I.J. Fair, "New error detection techniques and stopping criteria for turbo decoding," Canadian Conference on Electrical and Computer Engineering, pages 701-702, May 2000.

[15] B. Scanavino and G.M. Maggio, " A novel stopping criterion for turbo codes based on the average a posteriori entropy," In IEEE GLOBECOM conference, vol. 4, pp 2051-2055, December 2003.

[16] J. Heo and K. Chung and K. M. Chugg, "Simple stopping criterion for min-sum iterative decoding algorithm," IEEE Electronics Letters, vol. 37, pp. 1530-1531, December 2001.

[17] T. M. N. Ngatched and F. Takawira. "Simple stopping criterion for turbo decoding," IEEE Electronics Letters, vol. 37, pp. 1350-1351, October 2001.

[18] N. Y. Yu and M. G. Kim and Y. S. Kim and S. U. Chung, "Efficient stopping criterion for iterative decoding of turbo codes," IEEE Electronics Letters, vol. 37, pp. 73-75, January 2003.

[19] A. Worm and P. Hoeher, "Turbo-decoding without SNR estimation ," IEEE Communications Letters, pp 193-195, June 2000.

[20] Todd A. Summer and Stephen G. Wilson, "SNR Mismatch and Online Estimation in Turbo Decoding ," IEEE Transactions on Communications, Vol. 46, pp 421-423, April 1998.

[21] Wangrok Oh and Kyungwhoon Cheun, "Adaptive Channel SNR Estimation Algorithm for Turbo Decoder " IEEE Communications Letters, Vol. 4, pp 255-257, August 2000.

[22] H. El Gamal, A.R. Hammons Jr., "Analyzing the turbo decoder using the Gaussian approximation," IEEE Transaction on Information Theory, vol. 47, no. 2, February 2001. 